Paper

\title{
Optical Trap Detector with Large Acceptance Angle
}

\author{
Yoshiro ICHINO, Terubumi SAITO and Ichiro SAITO
}

\author{
National Metrology Institute of Japan, AIST
}

Received September 19, 2007, Accepted February 18, 2008

\begin{abstract}
We have developed a polarization-independent reflection-type silicon photodiode trap detector and characterized its performance by laser beam-based measurement. Three dimensional CAD-based modeling enables us to optimize its interior design, resulting in minimizing each distance between centers of adjacent photodiodes by rotating each photodiode by $45^{\circ}$ along each normal axis. It is expected by a simple ray-tracing simulation and also confirmed experimentally that the trap detector incorporating a photodiode with a large active area exhibits the largest acceptance angle ever proposed as the polarization-independent trap detector for the convergent incident beam. This is suitable for the national standard detector to realize and disseminate the cryogenic radiometer-based spectral power responsivity with high accuracy. It is also applicable to various kinds of working or transfer standard detectors for collimated or non-collimated monochromatic radiation. In addition, a history of development of trap detectors at national laboratories is reviewed.
\end{abstract}

\section{KEYWORDS: trap detector, silicon photodiode, spectral responsivity, acceptance angle}

\section{Introduction}

Silicon photodiodes are widely used as photodetectors for a variety of photometric and radiometric instruments owing to their outstanding advantages, such as high shunt resistance, low noise, high sensitivity, wide dynamic range and high internal quantum efficiency close to unity in the visible to near infrared (NIR) wavelength range. The national spectral responsivity scale based on a highaccuracy electrical substitution cryogenic radiometer ${ }^{11}$ has been established and disseminated at National Metrology Institute of Japan (NMIJ). The spectral responsivity calibration service for photodetectors in the ultraviolet (UV) - visible - NIR has been provided by NMIJ with minimum relative expanded uncertainty of $0.88 \%$ (a coverage factor of $k=2$ ) using monochromator-based comparator facility. The national photometric standards, that is, luminous intensity, luminous flux and illuminance, have been realized based on the standard photometer whose spectral responsivity is absolutely calibrated by the comparator.

A power-stabilized, profile-optimized laser beam is employed on the cryogenic radiometer-based absolute spectral power responsivity calibration facility. A nonnegligible interference effect should be taken into account as an additional source of uncertainty for the laser-based spectral responsivity calibration when a silicon photodiode covered with quartz or glass window is provided as a detector under test (DUT). A windowless photodiode is free from such interference effect and thus suitable for the laser-based calibration. Extreme care, however, should be taken to keep a bare silicon surface clean for maintaining the calibrated spectral responsivity value for a certain period in terms of quality assurance.

Such poor usability of windowless photodiodes becomes non-critical when they are assembled in a trap configuration, namely, aligned so as to "trap" the incident beam. Photodiodes incorporated in the trap detector are connected in parallel so as to output sum of their photocurrents. Although external efficiency of a single windowless photodiode is limited to $70 \%$ in the visible range in spite of internal quantum efficiency close to unity, sequential absorptions and reflections of the trapped light increase external quantum efficiency of the trap detector close to unity. As a consequence, its spectral responsivity is less sensitive to surface contamination compared with a single windowless photodiode. In addition, temperature dependence of spectral responsivity with its coefficient of an order of $10^{-4} /{ }^{\circ} \mathrm{C}$ for a single photodiode can be suppressed in a trap configuration. As far as applications for well-collimated beam are concerned, a trap detector with windowless photodiodes is suitable as a transfer standard or working standard detector to maintain calibrated spectral power responsivity value.

For non-collimated incident light, however, an acceptance angle of a trap detector should be considered as an additional issue of measurement uncertainty. Spectral responsivity value of DUT would be overestimated in the calibration if a convergent or divergent incident light 
would overfill one or more photodiodes in the trap detector with limited acceptance angle. Such an error may occur when the trap detector is employed to transfer the cryogenic radiometer-based absolute spectral responsivity value to the monochromator-based comparator for the spectral responsivity calibration of single photodiodes, because the output light from the monochromator is focused onto DUT by the mirror assembly so as to underfill its active area. A single photodiode, on the other hand, does not have any particular limitations concerning acceptance angle as far as its active area is underfilled by the incident light. Therefore, to minimize the uncertainty associated with transferring spectral responsivity value from collimated beam-based apparatus to non-collimated beam-based one, an acceptance angle of a trap detector should be designed as large as possible. A trap detector with a large acceptance angle is suitable not only for the transfer or working standard detector to establish national scales at NMIJ but also for high-accuracy laboratory standard detectors or even for consumer-level optical power meters to be used for a variety of incident geometries.

NMIJ has provided high-accuracy calibration service of spectral power responsivity of optical detectors at several fixed laser wavelengths in the visible range $(458-633 \mathrm{~nm})$ with minimum relative expanded uncertainty of $0.04 \%$ ( $k$ $=2$ ) since 2005. This calibration directly employs the cryogenic radiometer-based absolute spectral responsivity calibration facility. The above mentioned trap detector with its large acceptance angle is also suitable as DUT for this calibration service to meet customers' various applications. Although a number of national metrology institutes have proposed so far a variety of trap detector designs ${ }^{2-8)}$, their acceptance angles have not still been sufficient for fulfilling these needs.

In this work, we have developed a trap detector with large acceptance angle by optimizing its interior design. Historical aspects of trap detectors as well as experimental results to support their advantages are also described in this paper.

\section{Historical overview of trap detectors}

The first concept of the reflection-type trap detector was proposed by Zalewski et al. in $1983^{22}$. Their prototype detector contained four silicon photodiodes with planar beam-path configuration as illustrated in Figure 1(a), which was followed by the first commercial model QED100 (UDT Inc.). In this configuration, the incident beam is absorbed and reflected at each photodiode successively until it emits from the entrance aperture. All of the reflections (seven times in total) but the third one occurs with identical polarization, resulting in polarization dependence of the spectral responsivity of the trap detector. In addition, the acceptable incident geometry is limited to

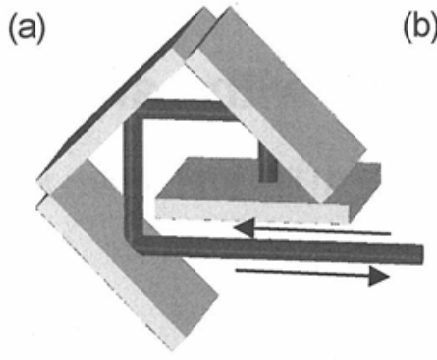

(b)

Figure 1 Configurations of trap detectors with planar

(a) and orthogonal (b) optical paths

Photodiodes and bidirectional beam paths are simply shown as plates and bending rods, respectively.

the collimated beam or the convergent or divergent light with its angle of a few degrees. Later, a new three-element configuration with nonplanar beam-path configuration was proposed by Fox et al. in 1991, followed by the second generation commercial trap detector QED-200 (UDT Inc.) as shown in Figure 1(b). Due to its orthogonal optical path of inner reflections, its spectral responsivity is theoretically polarization independent, although misalignment of each photodiode may induce unexpected polarization dependence ${ }^{3)}$. Each commercial model, however, incorporates three pieces of inversion-layer silicon photodiodes, which limits its both dynamic and spectral range. Fox et al. proposed a new trap detector whose external quantum efficiency is close to unity in the whole visible wavelength range, by applying $p^{-o n}-n$ type silicon photodiode (Hamamatsu S1337) instead of the threeelement orthogonal configuration ${ }^{4}$. Currently, a number of national metrology institutes have developed and maintained their own national standard trap detectors similar to this model. Alternative configurations of reflection-type trap detectors have also been proposed ${ }^{5}$.

Another fundamental concept of "trapping" incident light by multiple reflections on photodiodes is a "transmission" configuration ${ }^{6 \cdot-8)}$. The spectral responsivity of the six-element transmission trap detector with

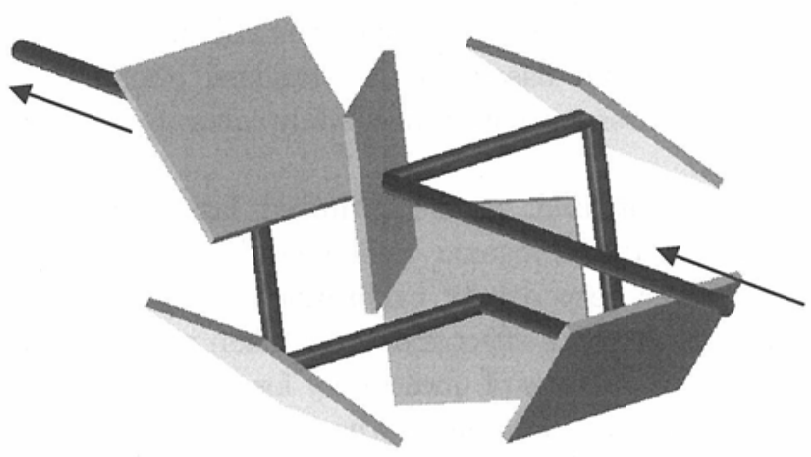

Figure 2 Configuration of six-element transmission trap detectors with an orthogonal optical path Photodiodes are shown in plates. 
orthogonal beam path (Figure 2) is polarization independent. In addition, the residual, untrapped output light is collinearly emitted from the exit port at the opposite side of the entrance port and thus easily visible to be useful for orientation alignment. This is in contrast with the reflection-type trap detector, which inherently emits retro-reflecting residual light.

Eppeldauer et al. developed a "tunnel trap" transmission-type detector with non-orthogonal, tornadolike optical path. This unconventional trap configuration allows larger acceptance angle compared with the conventional transmissiontype trap detectors with orthogonal optical paths by employing three large-area photodiodes together with three normal-size photodiodes ${ }^{7}$. The tunnel trap is optimized as an irradiance-mode radiometer with a precision aperture at the entrance port. Another approach proposed by Kübarsepp et al. to expand an acceptance angle of the transmission trap detector for irradiance-mode measurements is to minimize the distance between photodiodes, defined as that between centers of the active areas of adjacent photodiodes, by rotating each element by $45^{\circ}$ along each normal axis 8 .

The acceptance angles of these transmission-type trap detectors, however, are inherently too small to accept the convergent light with large convergence angle in the underfilled condition. The reflection-type configuration is more suitable for such application. Nevertheless, only few attempts have so far been made for further expanding the acceptance angle of the reflection-type trap detector.

\section{Modeling of trap detectors}

In this work, three dimensional (3D) CAD-based modeling was employed together with actual dimensions of commercial silicon photodiodes to optimize the interior design of the trap detector efficiently.

A three-element reflection-type trap detector (referred to as TD-3RA) was designed to expect the largest acceptance angle ever achieved as the polarizationindependent trap detector so as to accept tightly focused convergent light. A Hamamatsu S6337-01 silicon photodiode with an active area of $18 \times 18 \mathrm{~mm}$ was employed as the first photodiode (labeled as PD1 in Figure 3(a)). Both PD2 and PD3 are Hamamatsu S1337-1010 silicon photodiodes with active areas of $10 \times 10 \mathrm{~mm}$. To minimize the total optical path length from PD1 to PD3 for maximum acceptance angle of convergent incident light, we applied " $45^{\circ}$ rotation" concept proposed by Kübarsepp et al. ${ }^{8}$, namely, each photodiode is rotated by $45^{\circ}$ on each site along each normal axis. PD1-PD2 and PD2-PD3 distances are $14.4 \mathrm{~mm}$ and $10.1 \mathrm{~mm}$, respectively. All of the mounting blocks with photodiodes are packaged in a cylindrical case with diameter $d=50.4$ $\mathrm{mm}$ and total length $L=34.0 \mathrm{~mm}$ (Figure 3(c)).

We also modeled another reflection-type, " $45^{\circ}$ rotation" (a)

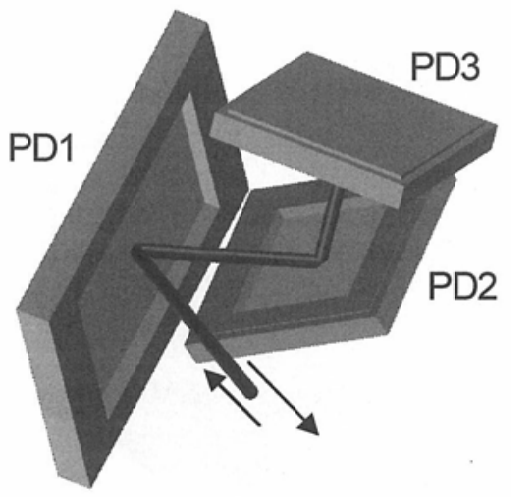

(b)

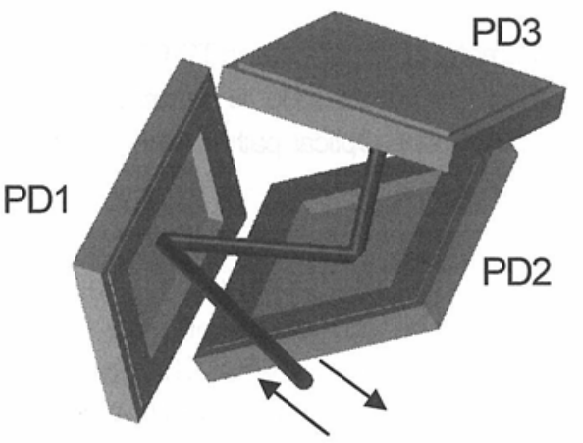

(c)

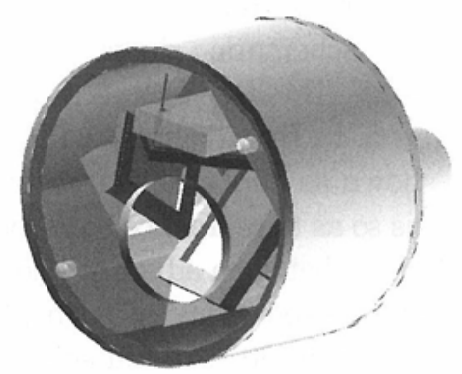

Figure 3 Photodiode geometries of TD-3RA (a) and TD-3RBa (b) Bidirectional optical paths are indicated as bending rods. PDn $(n=1-3)$ represents each photodiode in the incident order.

(c) Exterior design of TD-3RA

The front cover with entrance aperture is intentionally translucent to let the interior design visible.

concept trap detector (referred to as TD-3RBa) as a reference (Figure 3(b)). This is similar with TD-3RA, where S6337-01 photodiode is replaced by S1337-1010 for PD1. PD1-PD2 distance is found as $12.5 \mathrm{~mm}, 1.8 \mathrm{~mm}$ shorter than that of TD-3RA, while PD2-PD3 distance remains the same.

In addition to these reflection-type models, we remodeled the six-element transmission-type trap detector (referred to as TD-6TA) based on the model proposed by Kübarsepp et al.8) TD-6TA incorporates six S1337-1010 photodiodes, each rotated by $45^{\circ}$ along its normal axis as illustrated in Figure 4, in a 2-inch diameter cylindrical case $(L=42 \mathrm{~mm})$. Owing to the 3D-CAD modeling, we 


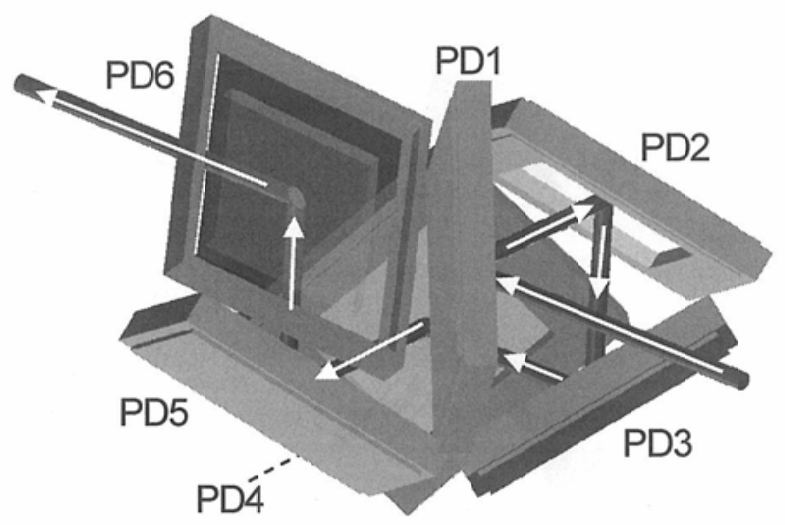

Figure 4 Geometry of photodiodes in TD-6TA

The last photodiode is illustrated intentionally as translucent. Optical path is also shown as bending rods with arrows.

succeeded in further optimizing the complicated construction of the transmission-type trap detector, resulting in each distance between the photodiodes of 12.0 $\mathrm{mm}, 1.0 \mathrm{~mm}$ reduction only between PD3-PD4.

\section{Estimation of acceptance angles}

We employ a schematic diagram to estimate acceptance angles of TD-3RA geometrically, which is indicated in Figure 5. In this diagram, sectional views of photodiodes are shown as bars and are aligned along the straight optical axis so as to reproduce each incident angle $\left(0^{\circ}\right.$ or $\left.45^{\circ}\right)$ and distance between adjacent photodiodes. We assume that spectral responsivity of each photodiode is completely uniform within $80 \%$ of full span of the active plane, for example, $8 \times 8 \mathrm{~mm}$ for $10 \times 10 \mathrm{~mm}$ active area. We defined it as an "effective" area. Unilluminated area shaded by the collar of the photodiode with respect to $45^{\circ}$ incident light is also taken into consideration as the outside of the effective area. The convergent incident light

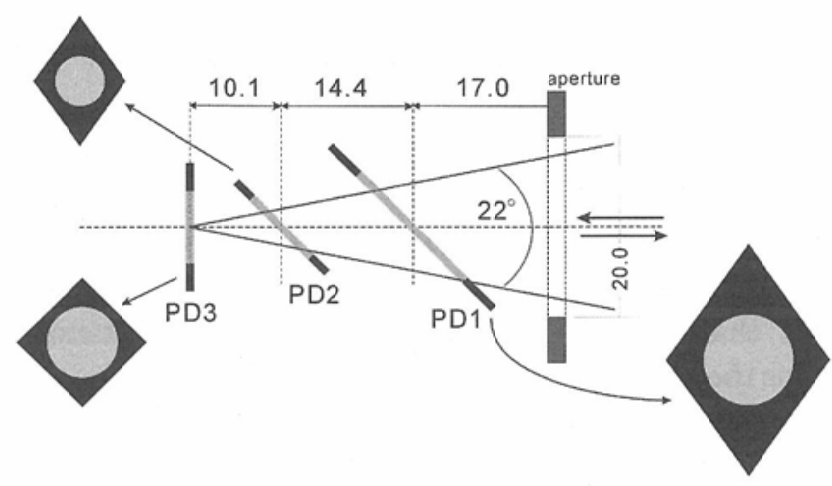

Figure 5 Schematic diagram of acceptance angle estimation for the trap detector TD-3RA

Active and effective areas are indicated as black (bars and rhombuses) and gray (bars and circles), respectively. is assumed to be focused onto the surface of PD3, reflected backward divergently and then emitted from the entrance aperture. For simplicity, we also assume that spatial profile of convergent light is circular and uniform.

The acceptance angle is defined as the largest angle of incidence to underfill the effective area. It is simulated as $22^{\circ}$ for TD-3RA as shown in Figure 5. Similarly, the acceptance angle of $15^{\circ}$ is obtained for TD-3RBa as illustrated in Figure 6. To investigate relationship in detail between size and geometry of photodiodes and the acceptance angle of the trap detector, we modeled another three configurations optimized for each photodiode size and geometry with the help of $3 \mathrm{D}-\mathrm{CAD}$ (referred to as TD$3 R B b, T D-3 R B c$ and TD-3RBd). Figure 6 indicates that " $45^{\circ}$ rotation" of photodiodes always makes it possible to expand the acceptance angle regardless of the size of photodiodes. This is well explained by the reduction of mechanical interference by the rotation. It is also found in Figure 6 that the replacement of a small photodiode with a large one at PD2 or PD3 position does not result in expanding the acceptance angle. It is concluded that the estimated acceptance angle of $22^{\circ}$ for TD-3RA is larger

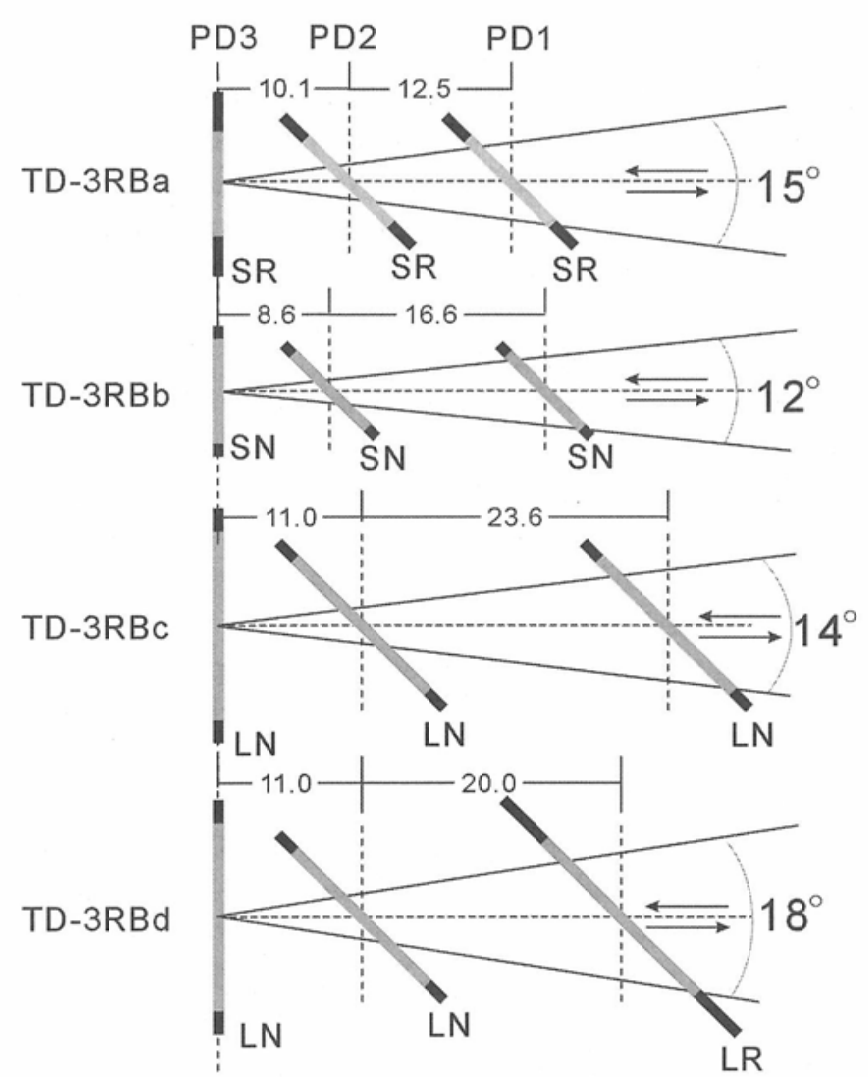

Figure 6 Comparison of estimated acceptance angles for various trap detector designs

SN: S1337-1010 with normal orientation, SR: S1337-1010 with $45^{\circ}$-rotated orientation, LN: S6337-01 with normal orientation, LR: S6337-01 with $45^{\circ}$-rotated orientation. Dimensions are given in $\mathrm{mm}$. 


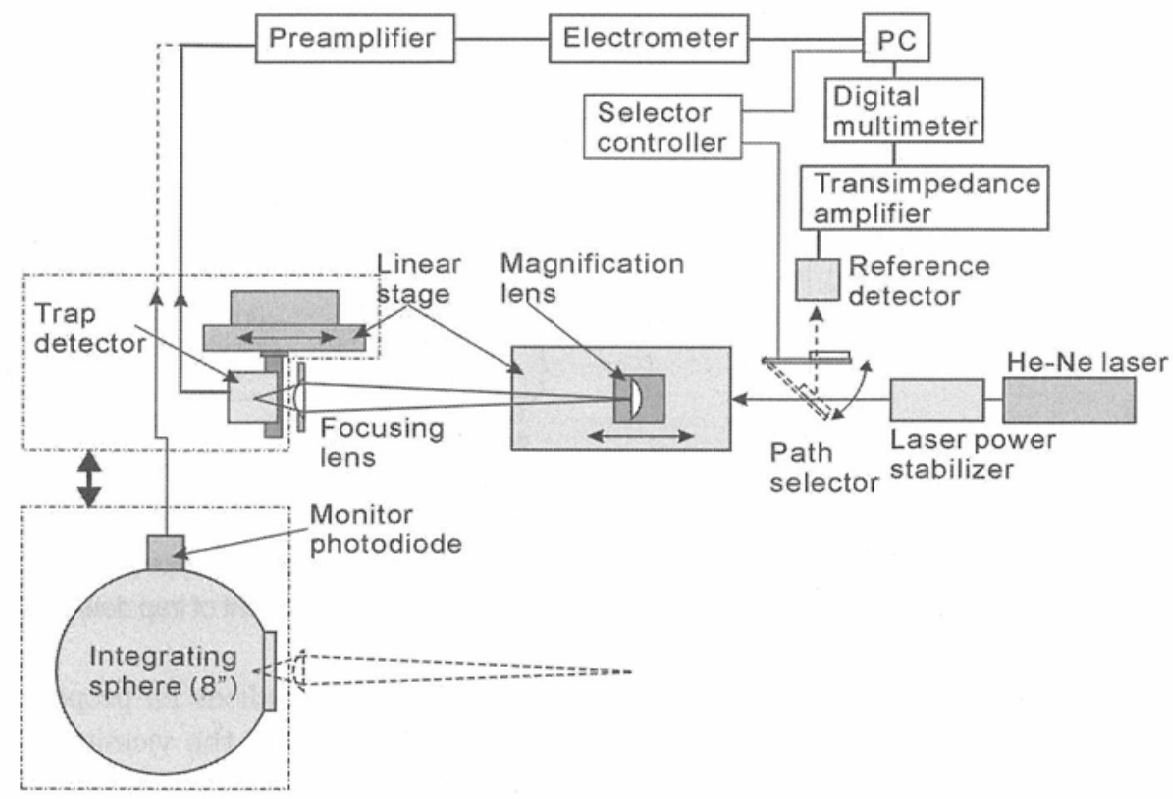

Figure 7 Measurement apparatus for verifying the acceptance angle of TD-3RA

than those for any other configurations modeled in this study. This large acceptance angle corresponds to the $\mathrm{F}$ number of 2.6, which is comparable to or even better than that of the compact $(\mathrm{f}<100 \mathrm{~mm})$ monochromator. For example, the spectral responsivity calibration facility at NMIJ incorporates a double-grating monochromator with a focal length of $250 \mathrm{~mm}$ and an F-number of 4.3. The acceptance angle of the TD-3RA is large enough to accept the monochromatic radiation from the monochromator through the 1:1 imaging optics. In contrast, this monochromatic convergent light may overfill the trap detector whose acceptance angle of smaller than $13^{\circ}$. As illustrated in Figure 6, an acceptance angle of a conventional polarization-independent reflection-type trap detector cannot achieve $12^{\circ}$. This indicates that the best measurement uncertainty on the absolute scale realization of the spectral responsivity can be reduced by replacing the current conventional trap detector with our new model TD-3RA as the transfer standard detector.

\section{Construction of trap detectors}

We assembled TD-3RA with windowless Hamamatsu S1337-1010 and S6337-01 silicon photodiodes, precisely manufactured aluminum blocks for mounting photodiodes, an aluminum cylindrical outer case and electrical wirings. Its experimental results are described in the following sections.

In addition, TD-3RBa was also assembled with the same parts as TD-3RA except windowless Hamamatsu S1337-1010 for PD1. As the large-area photodiode S633701 has smaller shut resistance and thus larger noise level compared with S1337-1010, we consider TD-3RB to be suitable for transferring or maintaining the calibrated value of spectral power responsivity with minimum calibration and measurement uncertainty for collimated or loosely convergent beam rather than tightly focused convergent light with large convergence angle. Accordingly, we do not describe the experimental results of TD-3RBa in this paper. The rest of the TD-3RBx family is not actually assembled in this study.

We also assembled the transmission-type TD-6TA as a prototype. This is the first home-built transmission-type polarization-independent trap detector in NMIJ. Due to its complicated construction, the number of national metrology institutes which have developed their own transmission-type trap detectors is limited.

\section{Experimental confirmation of acceptance angle}

To confirm the acceptance angle of TD-3RA obtained by the simulation in the previous section, we experimentally examined its response to the convergent incident light by employing the measurement apparatus as illustrated in Figure 7. A power-stabilized $\mathrm{He}-\mathrm{Ne}$ laser was employed as a light source. The power stability was further confirmed by measuring the optical power by the reference detector. An objective lens and a single plano-convex lens were used to magnifying and focusing the laser beam, respectively. The convergent light was applied to the trap detector so as to underfill its entrance aperture. The convergence angle was tunable by changing the distance between two lenses. The output current of the trap detector was measured by an electrometer equipped with a preamplifier. The objective lenses with its focal lengths of $25.4 \mathrm{~mm}$ and 16.2 $\mathrm{mm}$ were used for smaller $\left(2.5-11.5^{\circ}\right)$ and larger (13.4 $21.7^{\circ}$ ) convergence angles, respectively. We employed a linear translation stage to adjust the position of PD3 in DUT against the focal point of the convergent incident light. Deviation of PD3 position from the focal point 

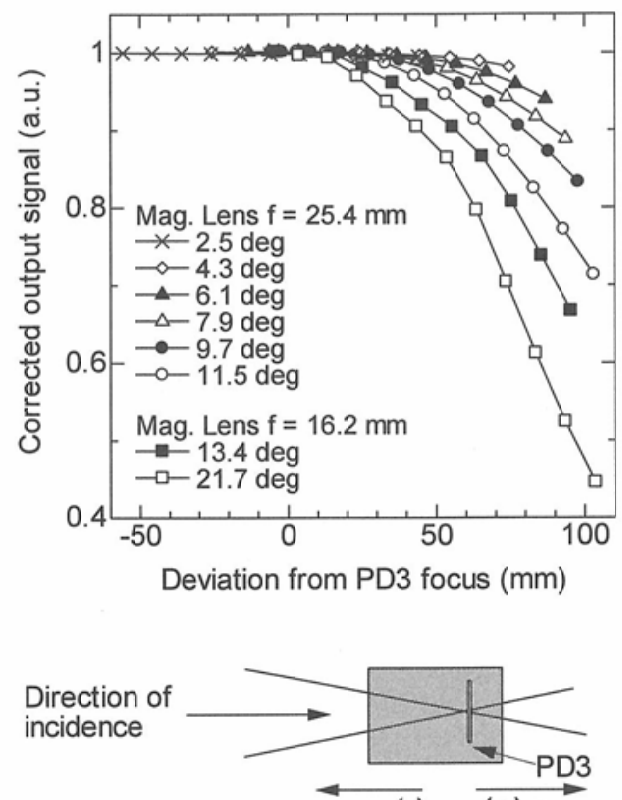

$(-) \quad \overline{(+)}$

Figure 8 Deviation dependence of the output signal of TD-3RA for convergent light with a variety of convergence angles

Signs of deviation are defined as illustrated above.

corresponds to the tolerance of adjustment for the convergent light. The deviation dependences of the response of TD-3RA with respect to a variety of the convergence angles from 2.5 to $21.7^{\circ}$ were experimentally obtained as shown in Figure 8. Each dependence curve was superimposed with one another without any normalization. Throughput of the lenses, which is determined by the amount of reflection or scattering loss on their surfaces and thus depends on the convergence angle of the incident light, should be taken into account as a correction factor for the output signals of the trap detector. To obtain this factor, we employed the integrating sphere attached with a monitor photodiode to be replaced with the trap detector as shown in Figure 8. The correction factor was determined as the ratio of the output of the photodiode for the convergent light with lenses against that for the collimated beam without lenses.

As shown in Figure 8, the tolerance of the focal point onto PD3 of TD-3RA for the convergent light is found to be no less than $20 \mathrm{~mm}$ for convergence angle smaller than $10^{\circ}$. When TD-3RA is employed as the transfer standard detector to realize the absolute scale of the spectral responsivity, the larger tolerance of the focal point can reduce the uncertainty relating to the alignment of the trap detector. The tolerance becomes smaller as the convergence angle becomes larger. And, what is more important, we can confirm experimentally that the convergent light even with the angle of close to $22^{\circ}$

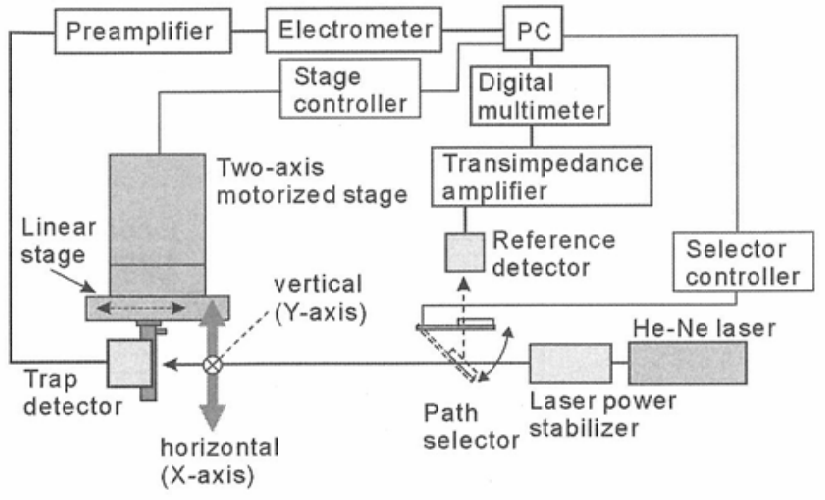

Figure 9 Experimental apparatus for spatial uniformity measurement of trap detectors

underfills each photodiode for proper operation, though its tolerance is limited to the vicinity of the surface of PD3. This result is well consistent with that obtained by the simulation using the simple schematic diagram, indicating that TD-3RA achieves the largest acceptance angle for the convergent light ever proposed.

\section{Spatial Uniformity}

We also examined the spatial uniformity of TD-3RA, which is important to transfer the spectral responsivity value calibrated with a collimated beam to that for applications using the convergent light, by employing the measurement apparatus depicted in Figure 9. This apparatus was equipped with two-axis motorized translation stage to scan TD-3RA horizontally and vertically against the incident beam. A circular $(\phi 2 \mathrm{~mm})$, intensity-stabilized beam from the $\mathrm{He}-\mathrm{Ne}$ laser was irradiated onto the detector without the entrance aperture. Scanning area was $12 \times 12 \mathrm{~mm}$ with a spacing of $0.5 \mathrm{~mm}$ for each axis, which is sufficient to cover the entire active area of the trap detector.

The spatial response of TD-3RA obtained by means of

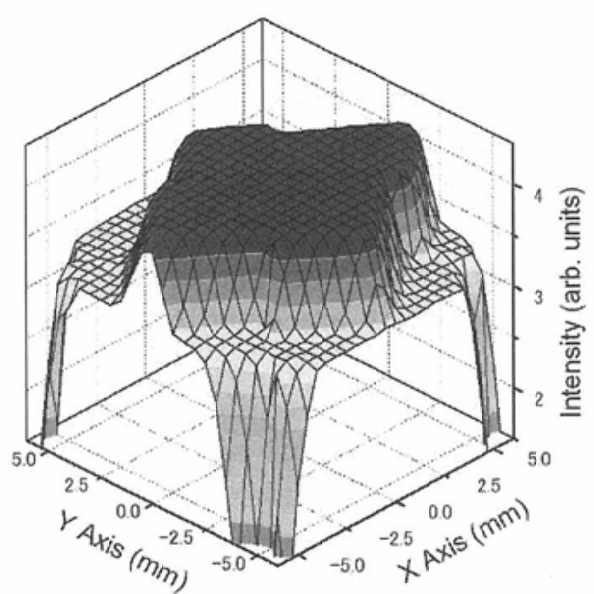

Figure 10 Spatial uniformity measurement for TD-3RA 
the laser scanning method is indicated in Figure 10. We clearly observed specific plateaus, the top plateau at the central area and the "hillside" around it. The central area exhibited the spatially uniform response with nonuniformity of less than $0.1 \%$. We used a calibrated power meter to estimate the absolute spectral responsivity of DUT and found that the external quantum efficiency of TD-3RA at the central top plateau was calculated as $\geq 0.995$. This indicates that TD-3RA ideally works as a three-element reflection trap detector at the central area. In addition, the relative responsivity of the "hillside" against the top plateau is found as approximately 0.8 . The step of the responsivity of 0.2 corresponds to the reflectance of the large PD1 (S6337-01) surface for the $p$-polarized incident beam at $\lambda=633 \mathrm{~nm}$. Namely, this indicates that the beam incident at the outer area around the central active area is not absorbed by PD2 or PD3 but by PD1. Moreover, the center of projected active area of each photodiode is roughly identical, suggesting that each photodiode of TD-3RA is aligned properly along the beam path. In conclusion, TD3RA is experimentally verified as the large acceptanceangle trap detector with excellent fundamental properties.

\section{Conclusions}

In this study, we have developed the polarizationindependent, reflection-type trap detector with large acceptance angle with the help of the 3D-CAD modeling software. Each distance between centers of adjacent photodiodes in a trap detector has been able to be reduced by rotating a photodiode by $45^{\circ}$ along its normal axis to avoid mechanical interference, expanding the acceptance angle of trap detectors. In conjunction with using a largearea photodiode as the first photoreceiver element, the trap detector TD-3RA is expected to have the largest acceptance angle of $22^{\circ}$ ever proposed, which is confirmed by means of the simple simulation method with the schematic diagram. By employing laser beam-based measurement apparatuses, we experimentally verified the large acceptance-angle of TD-3RA. We conclude that TD3RA is suitable not only for the national standard detectors to realize, transfer and maintain the spectral responsivity scale, but also high-accuracy laboratory standard detectors to be utilized for a wide variety of optical geometry, regardless of collimating condition, even probable for tightly focused output of the monochromator.

It should be noted that each photodiode are not still thoroughly aligned for ideal operation. It is well known that the spectral responsivity and its polarization dependence are particularly sensitive to misalignment of each photodiode ${ }^{9 \cdot 11}$. Off-centered alignment of each photodiode can induce unexpected vignetting error, resulting in the reduction of the spectral responsivity for convergent incident light. Polarization dependence as well as spatial uniformity should be evaluated individually.

\section{References}

(1) Ichino, Y. and Saito, I.: Improved Cryogenic Radiometry, Proceedings of the 25th Session of the CIE, Vol.1, pp.D2 18-21 (2003).

(2) Zalewski, E.F. and Duda, C.R.: Silicon photodiode device with $100 \%$ external quantum efficiency, Appl. Opt., 22, pp.2867-2873 (1983).

(3) Goebel, R., Yilmaz, S. and Pello, R.: Polarization dependence of trap detectors, Metrologia, 33, pp.207213 (1996).

(4) Fox, N. P: Trap Detectors and their Properties, Metrologia, 28, pp.197-202 (1991).

(5) Palmer, J. M.: Alternative Configurations for Trap Detectors, Metrologia, 30, pp.327-333 (1993).

(6) Gardner, J. L.: A fourelement transmission trap detector, Metrologia, 32, pp.469-472 (1995/96).

(7) Eppeldauer, G. P. and Lynch, D. C.: Opto-Mechanical and Electronic Design of a Tunnel-Trap $\mathrm{Si}$ Radiometer, J. Res. Natl. Inst. Stand. Technol., 105, pp.813-828 (2000).

(8) Kübarsepp, T., Kärhä, P. and Ikonen, E.: Characterization of a polarization-independent transmission trap detector, Appl. Opt., 36, pp.28072812 (1997).

(9) Goebel, R. and Stock, M: Nonlinearity and polarization effects in silicon trap detectors, Metrologia, 35, pp.413-418 (1998).

(10) Stock, K. D. and Heine, R.: Influence of vignetting errors on the relative spectral responsivity of trap detectors, Metrologia, 35, pp.447-450 (1998).

(11) Campos, J., Corredera, P., Pons, A., Corróns, A. and Fontecha J.L.: Reflectance dependencies of silicon trap detectors, Metrologia, 35, pp.455*460 (1998). 